\title{
The cardiothoracic ratio and all-cause and cardiovascular disease mortality in patients undergoing maintenance hemodialysis: results of the MBD-5D study
}

\author{
Hiroaki Ogata ${ }^{1}$ (D) Junji Kumasawa ${ }^{2,3,4}$ - Shingo Fukuma ${ }^{2,4,5} \cdot$ Masahide Mizobuchi $^{6}$ • \\ Eriko Kinugasa $^{1} \cdot$ Masafumi Fukagawa $^{7} \cdot$ Shunichi Fukuhara $^{2,5} \cdot$ Tadao Akizawa $^{6}$
}

Received: 5 July 2016 / Accepted: 4 January 2017 / Published online: 15 May 2017

(c) The Author(s) 2017. This article is an open access publication

\begin{abstract}
Background The cardiothoracic ratio (CTR) is a non-invasive left ventricular hypertrophy index. However, whether CTR associates with cardiovascular disease (CVD) and mortality in hemodialysis (HD) populations is unclear.

Methods Using a Mineral and Bone disorder Outcomes Study for Japanese CKD Stage 5D Patients (MBD-5D Study) subcohort, 2266 prevalent HD patients (age 62.8 years, female $38.0 \%$, HD duration 9.4 years) with secondary hyperparathyroidism (SHPT) whose baseline CTR had been recorded were selected. We evaluated associations between CTR and all-cause death, CVD death, or composite events in HD patients.

Results CTR was associated significantly with various background and laboratory characteristics. All-cause death, CVD-related death, and composite events increased across the CTR quartiles (Q). Adjusted hazard risk (HR) for all-cause death was 1.4 (95\% confidential interval, 0.9-2.1) in Q2, 1.9
\end{abstract}

The original version of this article was revised due to a retrospective Open Access order.

Electronic supplementary material The online version of this article (doi:10.1007/s10157-017-1380-2) contains supplementary material, which is available to authorized users.

Hiroaki Ogata

ogatah@med.showa-u.ac.jp

1 Department of Internal Medicine, Showa University Northern Yokohama Hospital, Chigsaki-chuo 35-1, Tsuzuki, Yokohama, Kanagawa 224-8503, Japan

2 Department of Healthcare Epidemiology, Graduate School of Medicine and Public Health, Kyoto University, Kyoto, Japan

3 Department of Critical Care Medicine, Sakai City Medical Center, Osaka, Japan
(1.3-2.9) in Q3, and 2.6 (1.7-4.0) in Q4, respectively (Q1 as a reference). The corresponding adjusted HR for CVD-related death was $1.8(0.8-4.2), 3.1$ (1.4-6.8), and 3.5 (1.6-7.9), and that for composite outcome was 1.2 (1.0-1.6), 1.7 (1.3-2.2), and $1.8(1.5-2.3)$, respectively. Exploratory analysis revealed that there were relationships between CTR and age, sex, body mass index, comorbidity of CVD, dialysis duration, dialysate calcium level and intact parathyroid hormone, phosphorus, hemoglobin, and usage of phosphate binder.

Conclusion CTR correlated with all-cause death, CVD death, and composite events in HD patients with SHPT.

Keywords Cardiothoracic ratio - Cardiovascular disease . CKD-MBD $\cdot$ MBD-5D study $\cdot$ Hemodialysis

\section{Introduction}

Left ventricular hypertrophy (LVH) is a significant predictor of cardiovascular morbidity and mortality in patients undergoing long-term hemodialysis (HD) therapy [1-7]. Many factors in the uremic milieu, including hypertension, volume overloading, anemia, chronic kidney disease-mineral and bone

4 Institute for Health Outcomes and Process Evaluation Research (iHope International), Kyoto, Japan

5 Center for Innovative Research for Communities and Clinical Excellence (CIRC2LE), Fukushima Medical University, Fukushima, Japan

6 Division of Nephrology, Department of Medicine, Showa University School of Medicine, Tokyo, Japan

7 Division of Nephrology, Endocrinology and Metabolism, Tokai University School of Medicine, Kanagawa, Japan 
disorder (CKD-MBD), the oxidative state, and inflammation, have been implicated in LVH pathogenesis [6-8]. The cardiothoracic ratio (CTR) is a readily available and non-invasive tool with which to assess the volume status and cardiomegaly. In addition, the CTR was found to correlate independently with $\mathrm{LVH}$ and target organ damage in hypertensive patients [9]. The CTR is likely to be a predictor all-cause and cardiovascular disease (CVD) mortality in patients with mild-tomoderate heart failure [9-11] or those undergoing coronary angiography [12]. However, it remains unclear that a higher CTR is associated with increased mortality among a hemodialysis-treated population [13-16]. This study was designed to evaluate whether CTR could predict all-cause death, CVD death, and the combined outcome of all-cause death and CVD-relate hospitalization in long-term HD patients, using data from the Mineral and Bone Disorder Outcomes Study for Japanese CKD Stage 5D patients (MBD-5D study) [17-21].

\section{Methods}

\section{Study design and patients}

The MBD-5D study was a 3-year prospective observational study involving hemodialysis patients with secondary hyperparathyroidism. The entire study cohort comprised 8229 enrolled patients, from whom a subcohort comprising 3276 patients was selected. The study involved relatively large dialysis facilities (each catering for $>100$ patients) in Japan, and patients were eligible for inclusion if they had consistently received hemodialysis for more than 3 months at the participating facilities as of January 1st, 2008, and if they met at least one of the following conditions: an intact parathyroid hormone (iPTH) level of $>180 \mathrm{pg} / \mathrm{ml}$ or receiving treatment with intravenous vitamin D receptor activators (VDRAs; calcitriol or maxacalcitol) or oral VDRA (falecalcitriol). Patients were excluded if they had undergone hemodialysis for less than 3 months at the time of evaluation for inclusion.

Details of the study design were reported previously [17, 18]. In this study, all patients whose baseline cardiothoracic ratio (CTR) had been recorded were enrolled.

\section{Exposure, outcomes, and covariates}

The CTR was measured by chest radiography in the postero-anterior view, while the patients were standing. The CTR was calculated by dividing the maximal horizontal heart breadth by the horizontal inner rib cage breadth. To confirm the accuracy of the measurements, a vertical line was drawn on each radiograph through the spinal midpoint from the sternum to the diaphragm. The maximum transverse cardiac diameter was obtained by adding the widest distance from the right heart border to the midline and the distance from the left cardiac border to the midline. At each facility, the CTR was measured at the start of the study and every 12 months throughout the study. Although the timing of CTR measurements was not clearly determined in the MBD-5D study, standard chest X-ray is performed immediately before the first HD session in a week in most Japanese dialysis facilities [22-24].

Patients were classified into four groups based on the baseline CTR quartiles: $\leq 46.8,46.8,<-50,50,<-53.6$, and $>53.6 \%$. As the CTR values changed during the study period, the CTR groups were expressed as time-varying covariates. It was permissible for patients to be placed in only one group at a time, although group placement during the study period changed according to the CTR.

The main outcome measure was all-cause death. The secondary outcome measures were cardiovascular disease (CVD)-related death and the combined outcome of allcause death and CVD-related hospitalization.

We also collected covariate demographic data (age, sex, BMI, smoking status, comorbidity of CVD and diabetes mellitus, and dialysis duration), baseline laboratory data (levels of iPTH, phosphorus, calcium, hemoglobin, albumin, and C-reactive protein), and covariate data related to dialysis (single-pool Kt/V and dialysate calcium level) and other medications (phosphate binder, vitamin D receptor activator, ACE inhibitor, and beta blocker) at the start of the study.

\section{Statistical methods}

Continuous variables were expressed as the mean \pm standard deviation, whereas categorical variables were expressed as a proportion. Continuous variables were compared among the groups using the analysis of variance (ANOVA). Categorical variables were compared using the Chi-squared test. We analyzed the adjusted hazard ratios (HRs) for all-cause death, CVD-related death, and the combined outcome of all-cause death and CVD-related hospitalization using the Cox proportional hazards model with the CTR level as a time-varying covariate. In the Cox proportional hazards model, missing CTR values other than the baseline value were replaced by the last observed CTR value. In all analyses, the CTR $\leq 46.8 \%$ group was used as a reference. All models were adjusted for all the covariates listed in Table 1 .

We also performed the same analysis stratified by the presence of DM and age ( $<64$ year, $>65$ year). We performed a sensitivity analysis using all the covariates as timevarying covariates. In this analysis, any missing covariates were replaced by the last observed values of those variables.

As an exploratory analysis, we performed multivariable regression analysis to investigate the relationship between CTR and covariates listed above. 
Table 1 Patient baseline characteristics

\begin{tabular}{|c|c|c|c|c|c|c|}
\hline Variable & $\begin{array}{l}\text { ALL } \\
(n=2266)\end{array}$ & $\begin{array}{l}\mathrm{Q} 1 \\
\mathrm{CTR} \leq 46.8 \% \\
(n=574)\end{array}$ & $\begin{array}{l}\text { Q2 } \\
\text { CTR } 46.8 \%<-50 \% \\
(n=596)\end{array}$ & $\begin{array}{l}\text { Q3 } \\
\text { CTR 50\%<-53.6\% } \\
(n=531)\end{array}$ & $\begin{array}{l}\mathrm{Q} 4 \\
\mathrm{CTR}>53.6 \% \\
(n=565)\end{array}$ & $P$ \\
\hline Age (year) & $62.8(12.9)$ & $58.3(13.1)$ & $61.6(12.1)$ & $63.7(12.1)$ & $67.6(12.3)$ & $<0.001$ \\
\hline Female (\%) & 38.0 & 27.0 & 29.5 & 41.1 & 55.0 & $<0.001$ \\
\hline Dialysis duration (year) & $9.4(8.2)$ & $9.0(8.0)$ & $9.2(7.6)$ & $9.9(8.3)$ & $9.8(8.8)$ & 0.171 \\
\hline CVD (\%) & 60.6 & 50.4 & 58.1 & 61.8 & 72.6 & $<0.001$ \\
\hline DM $(\%)$ & 34.6 & 34.7 & 35.7 & 34.3 & 33.6 & 0.896 \\
\hline Smoking & 10.7 & 12.9 & 10.7 & 10.0 & 9.2 & 0.213 \\
\hline BMI $\left(\mathrm{kg} / \mathrm{m}^{2}\right)$ & $21.3(3.6)$ & $21.4(3.0)$ & $21.7(3.3)$ & $21.3(4.0)$ & $20.8(3.8)$ & $<0.001$ \\
\hline $\mathrm{Kt} / \mathrm{V}$ & $1.4(0.3)$ & $1.4(0.3)$ & $1.4(0.3)$ & $1.4(0.3)$ & $1.4(0.3)$ & $<0.001$ \\
\hline $\mathrm{Ca}(\mathrm{mg} / \mathrm{dL})$ & $9.3(0.9)$ & $9.3(0.8)$ & $9.3(0.9)$ & $9.3(0.9)$ & $9.4(0.9)$ & 0.576 \\
\hline $\mathrm{P}(\mathrm{mg} / \mathrm{dL})$ & $5.5(1.4)$ & $5.5(1.4)$ & $5.6(1.4)$ & $5.5(1.3)$ & $5.5(1.4)$ & 0.824 \\
\hline iPTH (pg/mL) & $314.5(244.0)$ & $280.1(172.4)$ & $321.9(228.9)$ & $317.6(246.3)$ & $338.6(308.0)$ & $<0.001$ \\
\hline $\mathrm{Hb}(\mathrm{g} / \mathrm{dL})$ & $10.5(1.2)$ & $10.7(1.2)$ & $10.6(1.2)$ & $10.4(1.1)$ & $10.2(1.2)$ & $<0.001$ \\
\hline $\operatorname{ALB}(\mathrm{mg} / \mathrm{dL})$ & $3.7(0.4)$ & $3.8(0.4)$ & $3.8(0.3)$ & $3.7(0.4)$ & $3.6(0.4)$ & $<0.001$ \\
\hline CRP (mg/dL) & $0.5(1.4)$ & $0.5(1.6)$ & $0.4(1.5)$ & $0.3(0.7)$ & $0.6(1.5)$ & 0.030 \\
\hline VDRA use (\%) & 76.0 & 78.8 & 76.3 & 75.1 & 73.8 & 0.249 \\
\hline Phosphate binder use (\%) & 83.4 & 82.6 & 85.4 & 83.1 & 82.5 & 0.493 \\
\hline ACE inhibitor use (\%) & 7.7 & 5.9 & 8.1 & 10.4 & 6.7 & 0.034 \\
\hline ARB use $(\%)$ & 36.3 & 39.6 & 38.9 & 34.7 & 31.7 & 0.017 \\
\hline Beta blocker use (\%) & 6.1 & 6.3 & 8.4 & 4.9 & 4.8 & 0.037 \\
\hline Dialysate $\mathrm{Ca}(\mathrm{mEq} / \mathrm{L})$ & $2.7(0.2)$ & $2.7(0.2)$ & $2.7(0.2)$ & $2.7(0.2)$ & $2.7(0.3)$ & 0.113 \\
\hline
\end{tabular}

$C V D$ cardiovascular-related disease, $D M$ diabetes mellitus, $B M I$ body mass index, $C a$ calcium, $P$ phosphorus, ACE inhibitor angiotensin converting enzyme inhibitor, $A R B$ angiotensin receptor blocker

Differences were considered statistically significant at a two-sided $p$ value of $\leq 0.05$. All statistical calculations were performed using STATA version 13 (STATA, College Station, TX).

\section{Results}

\section{Patient characteristics}

In this study, 2266 patients were analyzed. In terms of the baseline CTR values, 574 patients were placed in the $\leq 46.8 \%$ group, 596 in the $46.8,<-50 \%$ group, 531 in the $50,<-53.6 \%$ group, and 565 in the $>53.6 \%$ group. Figure 1 shows a flow chart of the patients, and Table 1 shows the baseline clinical characteristics of the study population according to the CTR values at the start of the study. Table 1 shows the baseline clinical characteristics of the study population according to the CTR values at the start of the study. With regard to patient characteristics, the mean age was 62.8 years, the proportion of females was $38 \%$, and mean duration of dialysis was 9.4 years. Age, gender, CVD morbidities, body mass index, Kt/V, intact PTH, $\mathrm{Hb}$, albumin, $\mathrm{C}$-reactive protein, renin-angiotensin system

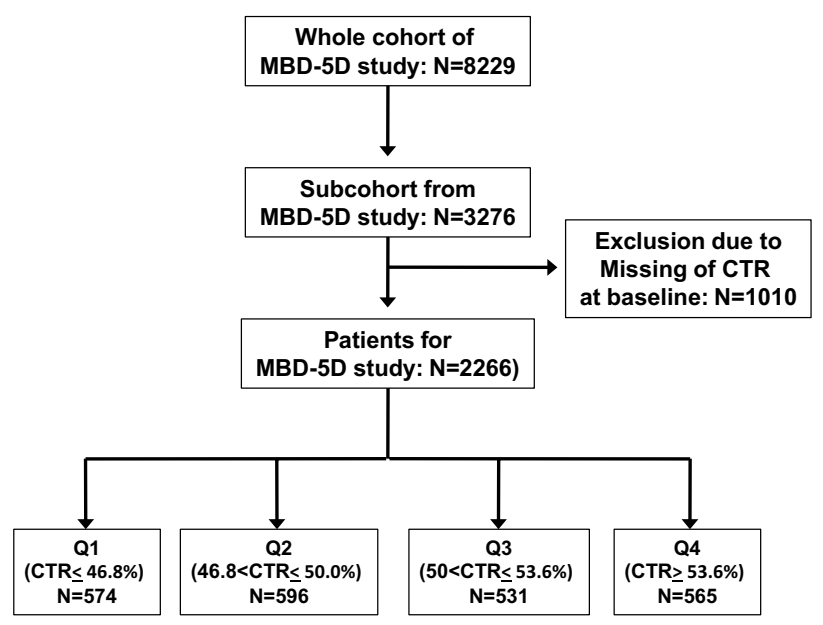

Fig. 1 Patient flow chart. Patients for the study subcohort were randomly selected from whole MBD-5D study cohort. Of the 3276 patients in the subcohort, 1010 were excluded due to missing CTR values at the baseline. Based on the CTR value at the baseline, 574, 596, 531, and 565 patients were placed into the CTR $\leq 46.8,46.8$, $<-50,50,<-53.6$, and $>53.6 \%$ groups, respectively. $M B D-5 D$ mineral and bone disorder outcomes study for Japanese chronic kidney disease stage $5 \mathrm{D}$ patients. CTR cardiothoracic ratio 
inhibitors use, and beta blockers use were significantly different among patients with each of the CTR quartiles.

\section{Association between CTR levels and mortality or composite outcome}

Of the 2266 patients, 404 (17.8\%) died due to any cause, 151 (6.7\%) died due to CVD-related events, and 835 composite events occurred during the study period. Table 2 shows the number of events that occurred and the proportion of events that occurred in the various CTR value groups.

Table 3a, $\mathrm{b}$ shows the unadjusted and adjusted HRs calculated using the Cox proportional hazards model with time-varying covariates. All-cause death, CVD-related death, and composite events increased as the CTR value increased.

Figures 2 and 3 show the subgroup analysis stratified by the presence of DM and age ( $<64,>65$ years). These analyses demonstrated the same pattern of associations with CTR and mortality.

\section{Sensitivity analysis}

Sensitivity analysis using all the covariates as time-varying covariates yielded results similar to those of the main analysis (Supplement).

\section{Exploratory analysis investigating the relationship between CTR and covariates}

Multivariable regression analysis showed that there were relationships between CTR and age, sex, BMI, comorbidity of CVD, dialysis duration, dialysate calcium level and level of iPTH, phosphorus, hemoglobin, and usage of phosphate binder (Table 4).

\section{Discussion}

CVD is more prevalent among HD patients and is the main cause of death $[25,26]$. Epidemiological studies have demonstrated that the CTR was a significant predictor of both all-cause and CVD-related mortality in patients regardless of undergoing dialysis therapy [10-16]. However, this study is a large scale, and is the first study to investigate the relationship between CTR measurement and clinical outcomes using a time-average Cox regression model in ESKD patients, focusing on CTR measurements as a timedependent covariate. Thus, the results of this study provide strong evidence that CTR corrected with mortality among dialysis population. The CTR measurement timing was thus more prolonged when compared with the timing used

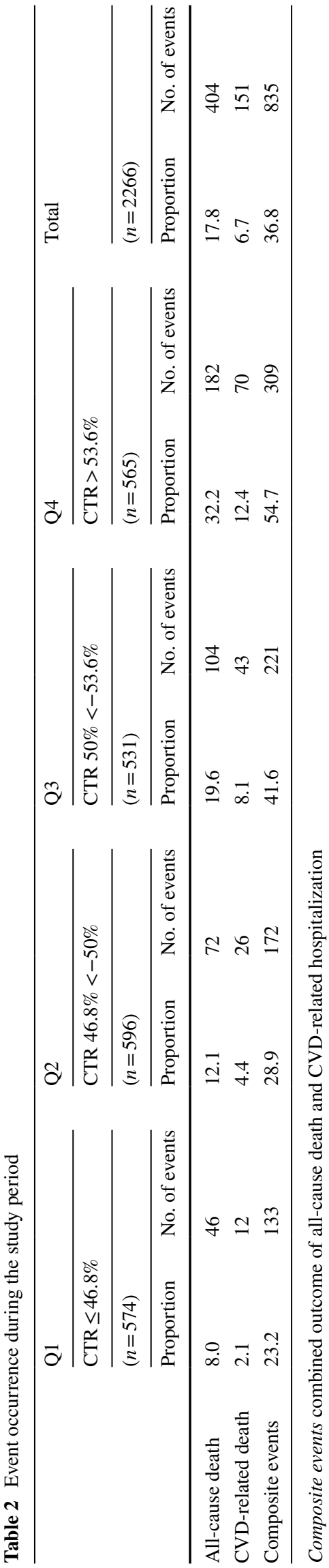


Table 3 Results of Cox proportional hazards model with time-varying covariates

\begin{tabular}{lllll}
\hline & $\begin{array}{l}\text { Q2 } \\
\text { CTR 46.8\%<-50\% } \\
\text { HR }(95 \% \text { CI })\end{array}$ & $\begin{array}{l}\text { Q3 } \\
\text { CTR 50\%<-53.6\% }\end{array}$ & $\begin{array}{l}\text { Q4 } \\
\text { CTR }>53.6 \%\end{array}$ & $\begin{array}{l}\text { Num- } \\
\text { ber of } \\
\text { patients }\end{array}$ \\
\hline (a) Unadjusted & & & & \\
All-cause death & $1.4(1.0-2.1)$ & $2.4(1.7-3.5)$ & $4.0(2.9-5.7)$ & 2266 \\
CVD-related death & $2.0(1.0-3.9)$ & $3.8(2.0-7.5)$ & $5.7(3.1-10.8)$ & 2266 \\
Composite events & $1.2(1.0-1.5)$ & $1.8(1.4-2.3)$ & $2.4(1.9-2.9)$ & 2266 \\
(b) Adjusted & & & & \\
All-cause death & $1.4(0.9-2.1)$ & $1.9(1.3-2.9)$ & $2.6(1.7-4.0)$ & 1954 \\
CVD-related death & $1.8(0.8-4.2)$ & $3.1(1.4-6.8)$ & $3.5(1.6-7.9)$ & 1954 \\
Composite events & $1.2(1.0-1.6)$ & $1.7(1.3-2.2)$ & $1.8(1.5-2.3)$ & 1954 \\
\hline
\end{tabular}

In these analyses, the CTR $\leq 46.8 \%$ group (Q1) was used as a reference

Composite events combined outcome of all-cause death and CVD-related hospitalization

${ }^{a}$ Unadjusted hazard ratio. CTR was treated as a time-varying covariate

${ }^{\mathrm{b}}$ Adjusted for age, sex, BMI, smoking status, comorbidity of CVD, diabetes mellitus, dialysis duration, levels of iPTH, phosphorus, calcium, hemoglobin, albumin and C-reactive protein, $\mathrm{Kt} / \mathrm{V}$, dialysate calcium level, phosphate binder, vitamin D receptor activator, ACE inhibitor, and beta blocker in other studies. In this study, we used a time-dependent Cox regression model to perform a detailed analysis of the associations between the CTR and the outcomes in a large subpopulation from the study patients of MBD-5D study [17-21], because the CTR measurements fluctuated in our large cohort study. After adjusting for various factors, higher CTR measurements were significantly associated with higher risks of all-cause and CVD mortality in this cohort. This study demonstrates that HRs for all-cause death, CVD-related death, and composite events (all-cause death and CVD-related hospitalization) increase across the CTR quartiles. In the subgroup analysis stratified by the presence of diabetes mellitus and age $(\leq 64, \geq 65$ year), there was seen the same pattern of association between CTR and mortality or the composite outcome. In an exploratory assessment using multivariable regression analysis, age, gender, body mass index, dry weight, CVD comorbidities, $\mathrm{Hb}, \mathrm{P}, \mathrm{iPTH}$, and P-binder use were significantly associated with CTR.

The CTR is an easy, reliable, and inexpensive tool for evaluating LVH and volume status without using specific equipment or technical modalities, such as ultrasonic echocardiography or magnetic resonance imaging (MRI). Furthermore, the CTR was found to independently correlate with the LV mass (LVM) and target organ damage [9]. The previous studies have shown that the CTR predicted systolic ventricular dysfunction and sudden death in patients with chronic heart failure $[11,27] . \mathrm{LVH}$ is an independent and strong predictor of all-cause and CVD mortality and CVD events in dialysis patients [1-7]. Many pathologic factors, including hypertension, volume overloading, local or systemic renin-angiotensin activation, sympathetic hyperactivity, inflammation, and oxidative stress, contribute to LVH in HD patients. In addition to classical factors, CKD-MBD also plays a crucial role in the LVH development $[6,7,28-30]$. Excess PTH and P overloading can induce cardiovascular remodeling in uremic animal models [31, 32]. Cardiac MRI, the most frequently used tool for cardiac remodeling assessments, was used to demonstrate that $\mathrm{Ca} \times \mathrm{P}$ products as well as the enddiastolic LV volume and pre-dialysis blood pressure levels could predict the LVM index in HD patients [33]. Recently, Yamamoto et al. reported an association between higher dietary $\mathrm{P}$ consumption and greater LVM in a communitybased multiethnic population without clinical CVD [34]. P overloading stimulates an increase in the serum fibroblast growth factor-23 levels and a decrease in 1,25-dihydroxyvitamin D production. These hormonal changes, which serve to maintain $\mathrm{P}$ homeostasis, have been implicated in cardiovascular remodeling in both experimental and observational studies 35-37. Interestingly, this study found that higher serum $\mathrm{P}$ levels and P-binder usage were associated with a higher CTR, which likely indicates increased LVH, in patients who were undergoing HD with SHPT. No previous studies have reported a significant association between $\mathrm{P}$ levels or P-binder usage and CTR values. Beyond doubt, the observational study never establishes any cause-andeffect conclusion. Further studies need to elucidate effect of P management on CTR measurements in CKD patients.

Agarwal demonstrated that hypervolemia, which is detected via relative plasma volume monitoring, could predict higher mortality among HD patients [38]. The CTR could significantly predict the volume status in HD patients. An observational study revealed that CTR-guided ultrafiltration management led to improvements in hypertension and cardiomegaly [39] and that a strict volume 
Fig. 2 Subgroup analysis stratified by the presence of diabetes mellitus (DM). Vertical line indicates the reference level. The adjusted HRs are shown with point estimates and 95\% confidence intervals. HRs were adjusted by age, sex, BMI, smoking status, comorbidity of CVD, diabetes mellitus, dialysis duration, levels of iPTH, phosphorus, calcium, hemoglobin, albumin and C-reactive protein, $\mathrm{Kt} / \mathrm{V}$, dialysate calcium level, phosphate binder, vitamin $\mathrm{D}$ receptor activator, ACE inhibitor, and beta blocker

\section{A All cause mortality}
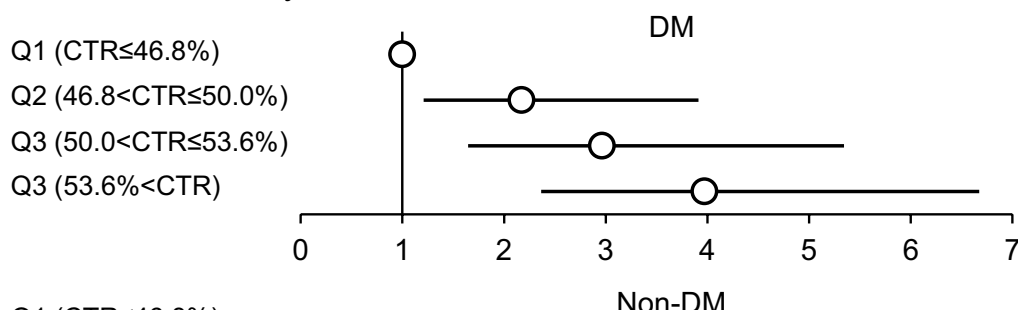

Reference

$2.17(1.21-3.91)$

$2.97(1.65-5.34)$

$3.97(2.36-6.67)$

Q1 (CTR $\leq 46.8 \%)$

Q2 (46.8<CTR $\leq 50.0 \%)$

Q3 $(50.0<$ CTR $\leq 53.6 \%)$

Q3 $(53.6 \%<C T R)$

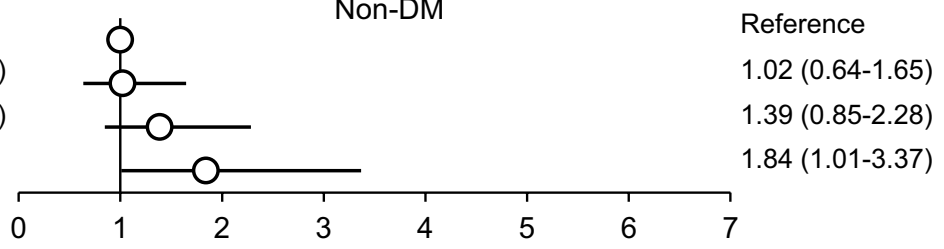

B CVD related mortality

Q1 $(C T R \leq 46.8 \%)$
Q2 $(46.8<C T R \leq 50.0 \%)$
Q3 $(50.0<$ CTR $\leq 53.6 \%)$
Q3 $(53.6 \%<$ CTR $)$

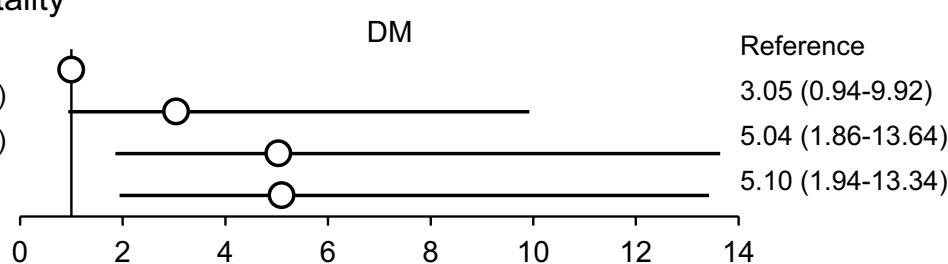

Q1 (CTR $\leq 46.8 \%)$

Q2 (46.8<CTR $\leq 50.0 \%)$

Q3 $(50.0<\mathrm{CTR} \leq 53.6 \%)$

Q3 $(53.6 \%<C T R)$

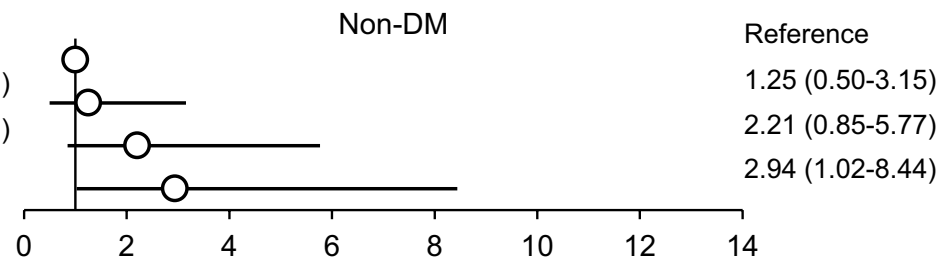

\section{All cause death and CVD related admission}

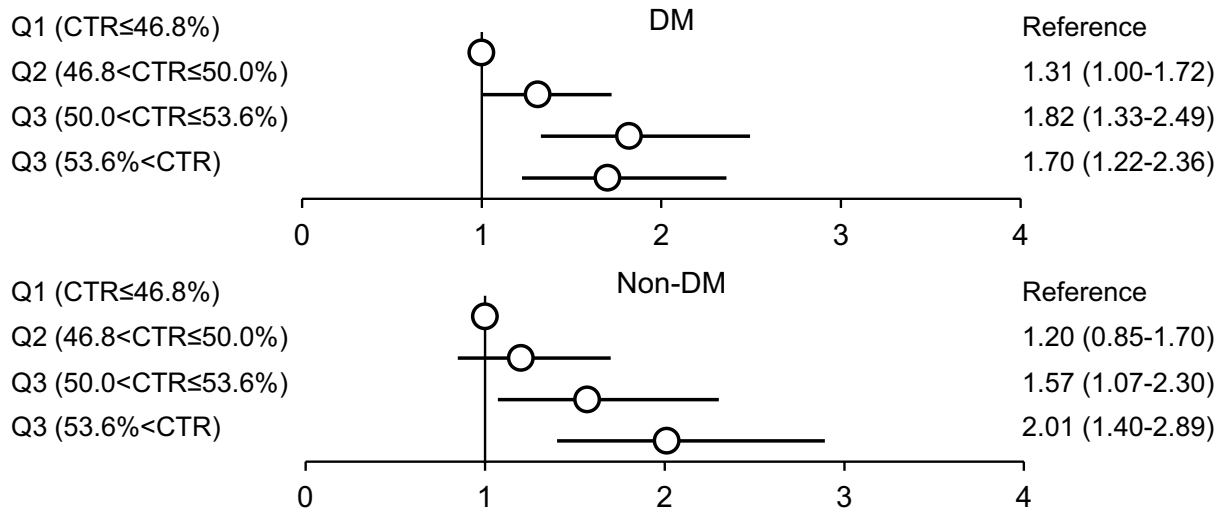

control strategy improved survival and reduced the CTR values even in patients undergoing the conventional HD treatment [40]. Therefore, the CTR is a useful volume status indicator in HD patients and can be modified through aggressive volume control management.

This study has several strengths and limitations with respect to interpretations of the results. First, given its observational nature, this study could not establish an obvious causal relationship between the CTR and clinical outcomes. Second, the study population was younger and had longer vintage of HD compared with general Japanese dialysis patients, although they were recruited from a large, multicenter cohort study in Japan (MBD-5D study). In addition, only the patients with SHPT were included in this study. Therefore, they might not have representability of general Japanese dialysis patients. Third, although it is very important whether the addition of CTR measurement to predictive model with patients' characteristics at baseline could be more predictive of mortality, time-dependent Cox regression model, which is used in this study, is 
Fig. 3 Subgroup analysis stratified by age $(<64$ year, $>65$ year). Vertical line indicates the reference level. The adjusted HRs are shown with point estimates and $95 \%$ confidence intervals. HRs were adjusted by age, sex, BMI, smoking status, comorbidity of CVD, diabetes mellitus, dialysis duration, levels of iPTH, phosphorus, calcium, hemoglobin, albumin and C-reactive protein, $\mathrm{Kt} / \mathrm{V}$, dialysate calcium level, phosphate binder, vitamin D receptor activator, ACE inhibitor, and beta blocker

A All cause mortality

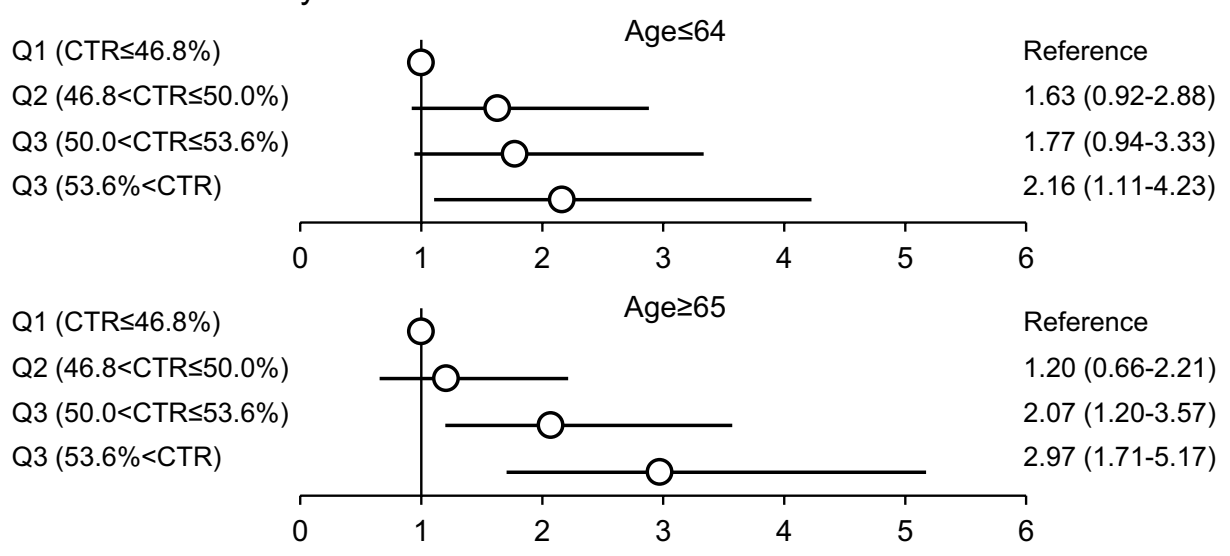

B CVD related mortality

Q1 $($ CTR $\leq 46.8 \%)$
Q2 $(46.8<C T R \leq 50.0 \%)$
Q3 $(50.0<C T R \leq 53.6 \%)$
Q3 $(53.6 \%<C T R)$

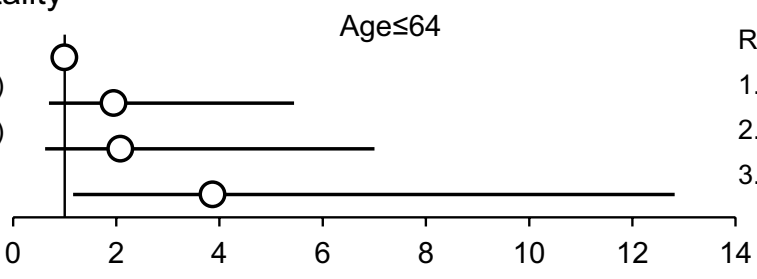

Reference $1.95(0.70-5.45)$ $2.09(0.62-7.00)$ $3.87(1.17-12.82)$

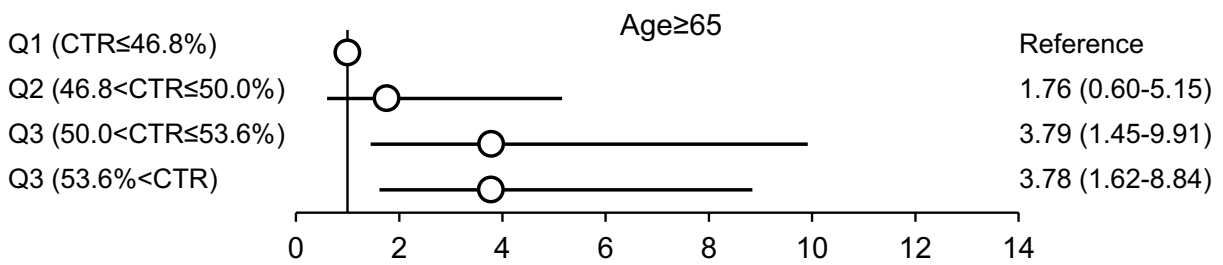

C All cause death and CVD related admission

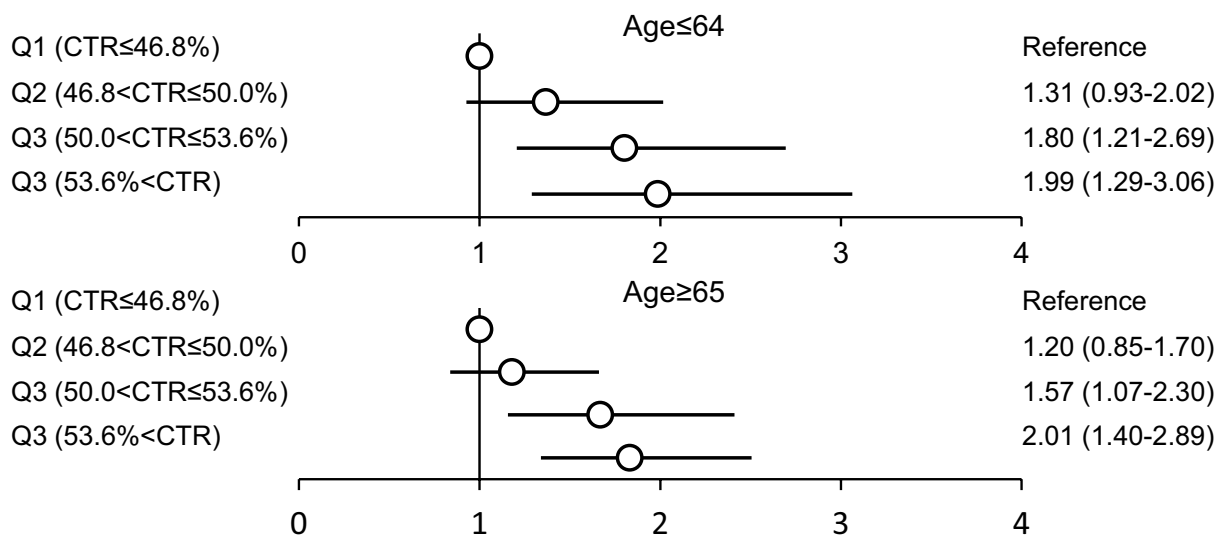

unsuitable for validating it. Bohn et al. reported that CTR was independently associated with mortality in retrospective cohort study of $824 \mathrm{HD}$ patients, but that CTR did not significantly improve predictive power for mortality when CTR was added to a base clinical model [16]. Hyperphosphatemia and P-binder usage were significant CTR determinants in this study population and were representative of CKD-MBD and SHPT. These results might also be specific to patients with established SHPT. However, a large number of patients with a very wide range of serum iPTH levels were carefully evaluated in this study; therefore, we believe that this limitation is negligible. Finally, arrhythmia (particularly atrial fibrillation) and heart valvular diseases, which are more prevalent in hemodialysis patients, can also contribute to higher CTR values. However, those factors were not assessed in detail in this study. Given these limitations, these results should be interpreted and generalized with caution.

This study has demonstrated that the CTR, which is associated with various clinical parameters, is significantly 
Table 4 Exploratory analysis on determinants of CTR

\begin{tabular}{llclr}
\hline Variable & Unit & $\beta$ coefficients & $95 \%$ CI & $P$ value \\
\hline Age & per 1 year & 0.093 & 0.070 to 0.117 & $<0.001$ \\
Sex, female & vs Male & 1.664 & $(1.040$ to 2.287$)$ & $<0.001$ \\
Diabetes mellitus comorbidity & vs No & -0.215 & $(-0.748$ to 0.318$)$ & 0.424 \\
Cardiovascular-related disease comorbidity & vs No & 1.176 & $(0.596$ to 1.756$)$ & $<0.001$ \\
Diabetes mellitus comorbidity & vs No & -0.215 & $(-0.748$ to 0.318$)$ & 0.424 \\
Smoking & vs No & 0.736 & $(-0.145$ to 1.618$)$ & 0.100 \\
Body mass index & per $1 \mathrm{~kg} / \mathrm{m}^{2}$ & 0.224 & $(0.079$ to 0.369$)$ & 0.003 \\
Urea clearance time/volume & per 0.1 & -0.602 & $(-1.872$ to 0.667$)$ & 0.348 \\
Dry weight & per $1 \mathrm{~kg}$ & -0.096 & $(-0.145$ to -0.046$)$ & $<0.001$ \\
Calcium & per $1 \mathrm{mg} / \mathrm{dL}$ & -0.409 & $(-0.341$ to 0.259$)$ & 0.786 \\
Phosphorus & per $1 \mathrm{mg} / \mathrm{dL}$ & 0.211 & $(0.025$ to 0.398$)$ & 0.027 \\
Intact parathyroid hormone & per $100 \mathrm{pg} / \mathrm{mL}$ & 0.002 & $(0.001$ to 0.003$)$ & $<0.001$ \\
Hemoglobin & per $1 \mathrm{~g} / \mathrm{dL}$ & -0.300 & $(-0.528$ to -0.073$)$ & 0.010 \\
Albumin & per $1 \mathrm{~g} / \mathrm{dL}$ & -0.342 & $(-1.013$ to 0.329$)$ & 0.313 \\
C-reactive protein & per $1 \mathrm{mg} / \mathrm{dL}$ & 0.046 & $(-0.117$ to 0.209$)$ & 0.576 \\
Vitamin D receptor activator & vs No & 0.312 & $(-0.525$ to 0.588$)$ & 0.911 \\
Phosphate binder & vs No & 0.746 & $(0.072$ to 1.420$)$ & 0.031 \\
Angiotensin converting enzyme inhibitor & vs No & 0.145 & $(-0.729$ to 1.019$)$ & 0.741 \\
Beta blocker & vs No & -0.068 & $(-1.317$ to 1.181$)$ & 0.914 \\
\hline
\end{tabular}

predictive of all-cause and CVD mortality, and composite outcomes in patients receiving HD. These results suggest that efforts of reduced CTR or prevention of its enlargement, including an aggressive volume control strategy, optimal anemia, and CKD-MBD management, could potentially reduce the CVD morbidity and mortality rates in HD patients.

Acknowledgements We would like to thank the following MBD-5D study advisory investigators: Masashi Suzuki (Shinrakuen Hospital), Yoshindo Kawaguchi (Shiomidai Hospital), Akira Saito (Shonan Tobu Sougo Hospital), Yoshiki Nishizawa (Osaka City University Graduate School of Medicine), Yusuke Tsukamoto (Itabashi Chuo Medical Center), Satoshi Kurihara (Tsukinomori Clinic), Takashi Akiba (Tokyo Women's Medical University), Yuzo Watanabe (Kasugai Municipal Hospital), Yoshihiro Tominaga (Nagoya Daini Red Cross Hospital), Takashi Shigematsu (Wakayama Medical University), Masaaki Inaba (Osaka City University Graduate School of Medicine), Jun Minakuchi (Kawashima Hospital), Hideki Hirakata (Fukuoka Red Cross Hospital), Keitaro Yokoyama (The Jikei University School of Medicine), Naoki Kimata (Tokyo Women's Medical University), Fumihiko Koiwa (Showa University Fujigaoka Hospital), Ryoichi Ando (Musashino Red Cross Hospital), Junichiro J. Kazama (Niigata University), Takatoshi Kakuta (Tokai University School of Medicine), Hirotaka Komaba (Tokai University School of Medicine), Daijo Inaguma (Nagoya Daini Red Cross Hospital), Eiji Ishimura (Osaka City University Graduate School of Medicine), Hideki Tahara (Osaka City University Graduate School of Medicine), Kazuhiko Tsuruya (Kyushu University), Akira Fujimori (Konan Hospital), and Ryo Kido (Inagi Municipal Hospital).

\section{Compliance with ethical standards}

The study protocol of MBD-5D study was approved by the central (Kobe University School of Medicine (No. 754)) and local ethical review boards. This study was an observational study using anonymized data collected during routine practice; informed consent was not mandatory according to the ethical guidelines for epidemiological research in Japan. The study was conducted in accordance with the principles of the Declaration of Helsinki and the ethical guidelines for epidemiological research in Japan, and it was registered at ClinicaiTrials.gov, No. NCT00995163.

Conflict of interest The MBD-5D study was supported by research grants from Kyowa Hakko Kirin without restrictions on publications. H.O. received honoraria from Kyowa Hakko Kirin. M.M. received honoraria from Kyowa Hakko Kirin. M.F. has served as a consultant for Kyowa Hakko Kirin, received honoraria from Kyowa Hakko Kirin, and received grants (research support) from Kyowa Hakko Kirin. T.A. has served as a consultant and advisor of Kyowa Hakko Kirin, and is a member of a Kyowa Hakko Kirin speakers' bureau. S.F. has served as a scientific advisor for Kyowa Hakko Kirin and received grants (research support) from Kyowa Hakko Kirin.

Open Access This article is distributed under the terms of the Creative Commons Attribution 4.0 International License (http://creativecommons.org/licenses/by/4.0/), which permits use, duplication, adaptation, distribution and reproduction in any medium or format, as long as you give appropriate credit to the original author(s) and the source, provide a link to the Creative Commons license and indicate if changes were made.

\section{Appendix}

We would like to thank the following MBD-5D study investigators who participated in this study: Nobuo Hashimoto (H.N.MEDIC), Mari Ishida (Kitasaito Hospital), Toshiyuki Date (Date Clinic), Kiyotaka Yabuki (Yabuki Hospital), Hideki Tanida (Tendo Onsen Yabuki 
Clinic), Fumitoshi Yamauchi (San-ai Hospital), Mikihiko Fujishima (Yahaba Clinic), Tomohito Matsunaga (Eijinkai Hospital), Jun Urae (Ishinomaki Clinic), Hiroshi Kawaguchi (Iwaki Urological Hospital), Ikuo Takahashi (Kisen Hospital), Yoshiko Tanaka (Shinjuku Ishikawa Clinic), Hideo Kobayashi (Suda Clinic), Maki Takahashi (Suda Naika Clinic), Tatsuya Nonaka (Seishokai Memorial Hospital), Hideto Emoto (Tokai Hospital), Kyosuke Nishio (Shinkoiwa Clinic), Atsushi Hayama (Moriyama Rehabilitation Hospital), Toshio Shinoda (Kawakita General Hospital Dialysis Center), Takashi Kono (Mihama Narita Clinic), Takahiro Mochizuki (Kameda Medical Center), Yasuo Kimura (Shin-kashiwa Clinic), Noriyoshi Murotani (Chiba Social Insurance Hospital), Satoshi Yamaguchi (Asahi Hospital), Taichi Nakanishi (Kurihama Clinic), Kiyoshi Ozawa (Yokosuka Clinic), Takashi Nagaoka (Sagamihara Clinic), Takao Suga (Bousei Hiratsuka Clinic), Masakazu Suda (Suda Medical Clinic), Yoshikazu Goto (Saiyu Soka Hospital), Michio Kuwahara (Shuwa General Hospital Hemodialysis Clinic), Hiromi Shimoyama (Yuai Clinic), Kimihiko Matsuyama (Misato Kenwa Clinic), Kazue Ueki (Toho Hospital), Kyoko Ito (Heisei Hidaka Clinic), Katsuhiko Miyamoto (Seseragi Hospital), Takashi Ishizu (Tukuba Central Hospital), Shuichi Kikuchi (Ohba Renal Clinic), Masaki Kobayashi (Tokyo Medical University Ibaraki Medical Center), Mitsuyoshi Furuhashi (Maruyama Hospital), Masanori Wakabayashi (Bousei Dai-ichi Clinic), Kazuyoshi Nakamura (Fujidaiichi Clinic), Hirotake Kasuga (Kaikoukai Central Clinic), Itsuo Yokoyama (Nagoya Memorial Foundation Narumi Clinic), Chikao Yamazaki (Masuko Clinic SUBARU), Kijun Nagata (Sawada Hospital), Yasumasa Kawade (Suzuka Kidney Clinic), Toshiaki Kawanaka (Ishikiriseiki Hospital), Yoshihiro Tsujimoto (Inoue Hospital), Mikio Okamura (Ohno Memorial Hospital), Shigeki Okada (Okada Clinic), Senji Okuno (Kidney Center Shirasagi Clinic), Harumi Nagayama (Nagayama Hemodialysis Clinic), Shuji Okazaki (Nagayama Hospital), Yoshinori Tone (Fujii Clinic), Ibuki Yajima (Ibuki Clinic), Kouji Shibuya (Sumiyoshigawa Hospital), Kunihiko Yoshiya (Hara Genitourinary Hospital), Morihiro Kondou (Otowa Kinen Hospital), Satoru Yamazaki (Tojinkai Hospital), Ryoichi Miyazaki (Fujita Memorial Hospital), Katsuhiko Arimoto (Shigei Medical Research Hospital), Misaki Moriishi (Nakajima Tsuchiya Clinic), Takahito Nasu (Tokuyama Central Hospital), Seiichi Obayashi (Kinashi Obayashi Hospital), Yuzuru Sato (Sato Junkankika Naika), Takao Tanaka (Ohji Hospital), Hidetoshi Nakamura (Kokura Daiichi Hospital), Nobuhiko Koga (Shin-Koga Clinic), Harumichi Higashi (St. Mary's Hospital), Kougi Yuu (Takahashi Naika Clinic), Asako Kitamura (Chikuho Social Insurance Hospital), Tomoji Matsumae (Murakami Memorial Hospital), Katsushige Abe (Jinikai Hospital),
Masahiro Kawatomi (Kawatomi Internal Medicine Clinic), Motoko Tanaka (Akebono Clinic), Chisa Nogami (Kumamoto Urological Hospital), Etsuo Yoshidome (Ikeda Hospital), Shinyu Miyagi (Okinawa Daiichi Hospital), Satoshi Nakazato (Chibana Clinic), Yoshiki Shiohira (Tomishiro Central Hospital), and Kiyoyuki Tokuyama (Tokuyama Clinic).

\section{References}

1. Silberberg JS, Barre PE, Prichard SS, Sniderman AD. Impact of left ventricular hypertrophy on survival in end-stage renal disease. Kidney Int. 1989;36:286-90.

2. Foley RN, Parfrey PS, Harnett JD, et al. The prognostic importance of left ventricular geometry in uremic cardiomyopathy. J Am Soc Nephrol. 1995;5:2024-31.

3. London GM, Pannier B, Guerin AP, et al. Alterations of left ventricular hypertrophy in and survival of patients receiving hemodialysis: follow-up of an interventional study. J Am Soc Nephrol. 2001;12:2759-67.

4. Zoccali C, Benedetto FA, Mallamaci F, et al. Prognostic impact of the indexation of left ventricular mass in patients undergoing dialysis. J Am Soc Nephrol. 2001;12:2768-74.

5. Zoccali C, Benedetto FA, Mallamaci F, et al. Left ventricular mass monitoring in the follow-up of dialysis patients: prognostic value of left ventricular hypertrophy progression. Kidney Int. 2004;65:1492-8.

6. Charytan D. Is left ventricular hypertrophy a modifiable risk factor in end-stage renal disease? Cur Opin Nephrol Hypertens. 2014;23:578-85.

7. Di Lullo L, Gorini A, Russo D, et al. Left ventricular hypertrophy in chronic kidney disease patients: from pathophysiology to treatment. Cardiorenal Med. 2015;5:254-66.

8. Silberberg JS, Rahal DP, Patton DR, Sniderman AD. Role of anemia in the pathogenesis of left ventricular hypertrophy in endstage renal disease. Am J Cardiol. 1989;64:222-4.

9. Rayner BL, Goodman H, Opie LH. The chest radiograph. A useful investigation in the evaluation of hypertensive patients. Am J Hypertens. 2004;17:507-10.

10. Giamouzis G, Sui X, Love TE, et al. A propensity-matched study of the association of cardiothoracic ratio with morbidity and mortality in chronic heart failure. Am J Cardiol. 2008;101:343-7.

11. Kearney MT, Fox KA, Lee AJ, et al. Predicting sudden death in patients with mild to moderate chronic heart failure. Heart. 2004;90:1137-43.

12. Zaman MJ, Sanders J, Crook AM, et al. Cardiothoracic ratio within the "normal" range independently predicts mortality in patients undergoing coronary angiography. Heart. 2007;93:491-4.

13. Chen KH, Lin-Tan DT, Huang WH, et al. Cardiothoracic ratio, malnutrition, inflammation, and two-year mortality in non-diabetic patients on maintenance hemodialysis. Kidney Blood Press Res. 2008;31:143-51.

14. Yen TH, Lin JL, Lin-Tan DT, Hsu KH. Cardiothoracic ratio, inflammation, malnutrition, and mortality in diabetes patients on maintenance hemodialysis. Am J Med Sci. 2009;337:421-8.

15. Chen KH, Hung CC, Lin-Tan DT, et al. Cardiothoracic ratio association with mortality in patients on maintenance peritoneal dialysis. Ther Apher Dial. 2011;15:81-8.

16. Bohn E, Tangri N, Gali B, et al. Predicting risk of mortality in dialysis patients: a retrospective cohort study evaluating the prognostic value of a simple chest X-ray. BMC Nephrol. 2013;14:263. 
17. Fukuhara S, Akizawa T, Fukagawa M, et al. Mineral and bone disorders outcomes study for Japanese chronic kidney disease stage 5D patients: rationale and study design. Ther Apher Dial. 2011;15:169-75.

18. Akizawa T, Kido R, Fukagawa M, Onishi Y, et al. Decreases in PTH in Japanese hemodialysis patients with secondary hyperparathyroidism: associations with changing practice patterns. Clin J Am Soc Nephrol. 2011;6:2280-8.

19. Fukagawa M, Fukuma S, Onishi Y, et al. Prescription patterns and mineral metabolism abnormalities in the cinacalcet era: results from the MBD-5D study. Clin J Am Soc Nephrol. 2012;7:1473-80.

20. Fukagawa M, Komaba H, Onishi Y, et al. Mineral metabolism management in hemodialysis patients with secondary hyperparathyroidism in Japan: baseline data from the MBD-5D. Am J Nephrol. 2011;33:427-37.

21. Fukagawa M, Kido R, Komaba $\mathrm{H}$, et al. Abnormal mineral metabolism and mortality in hemodialysis patients with secondary hyperparathyroidism: evidence from marginal structural models used to adjust for time-dependent confounding. Am J Kidney Dis. 2014;63:979-87.

22. Kawamura M, Mitsunobu S, Hisanaga S, et al. Incidence, outcome, and risk factors of cerebrovascular events in patients undergoing maintenance hemodialysis. Am J Kidney Dis. 1998;31:991-6.

23. Asakawa T, Joki N, Tanaka T, et al. Association between the hemoglobin level and cardiothoracic ratio in patients on incident dialysis. Cardiorenal Med. 2014;3:189-200.

24. Oosugi K, Fujimoto N, Dohi J, et al. Hemodynamic and pathophysiological characteristics of intradialytic blood pressure elevation in patients with end-stage renal disease. Hypertens Res. 2014;37:158-65.

25. Nakai S, Iseki K, Itami N, Ogata S, et al. An overview of regular dialysis treatment in Japan (as of 31 December 2010). Ther Apher Dial. 2012;16:483-521.

26. Wakasugi M, Kazama JJ, Yamamoto S, et al. Cause-specific excess mortality among dialysis patients: comparison with the general population in Japan. Ther Apher Dial. 2013;17:298-304.

27. Shah S, Davies MK, Cartwright D, Nightingale P. Management of chronic heart failure in the community: role of a hospital based open access heart failure service. Heart. 2004;90:755-9.
28. Ogata H, Koiwa F, Kinugasa E, Akizawa T. CKD-MBD: impact on management of kidney disease. Clin Exp Nephrol. 2007;11:261-8.

29. Achinger SG, Ayus JC. Left ventricular hypertrophy: is hyperphosphatemia among dialysis patients a risk factor? J Am Soc Nephrol. 2006;17:S255-61.

30. Achinger SG, Ayus JC. The role of vitamin D in left ventricular hypertrophy and cardiac function. Kidney Int Suppl 2005:S37-42.

31. Ogata H, Ritz E, Odoni G, et al. Beneficial effects of calcimimetics on progression of renal failure and cardiovascular risk factors. J Am Soc Nephrol. 2003;14:959-67.

32. Amann K, Ritz E, Wiest G, et al. A role of parathyroid hormone for the activation of cardiac fibroblasts in uremia. J Am Soc Nephrol. 1994;4:1814-9.

33. Patel RK, Oliver S, Mark PB, et al. Determinants of left ventricular mass and hypertrophy in hemodialysis patients assessed by cardiac magnetic resonance imaging. Clin J Am Soc Nephrol. 2009;4:1477-83.

34. Yamamoto KT, Robinson-Cohen C, de Oliveira MC, et al. Dietary phosphorus is associated with greater left ventricular mass. Kidney Int. 2013;83:707-14.

35. Faul C, Amaral AP, Oskouei B, et al. FGF23 induces left ventricular hypertrophy. J Clin Invest. 2011;121:4393-408.

36. Mirza MA, Larsson A, Melhus H, et al. Serum intact FGF23 associate with left ventricular mass, hypertrophy and geometry in an elderly population. Atherosclerosis. 2009;207:546-51.

37. Li YC, Kong J, Wei M, et al. 1,25-Dihydroxyvitamin D(3) is a negative endocrine regulator of the renin-angiotensin system. $\mathrm{J}$ Clin Invest. 2002;110:229-38.

38. Agarwal R. Hypervolemia is associated with increased mortality among hemodialysis patients. Hypertension. 2010;56:512-7.

39. Poggi A, Maggiore Q. Cardiothoracic ratio as a guide to ultrafiltration therapy in dialyzed patients. Int J Artif Organs. 1980;3:332-7.

40. Ozkahya M, Ok E, Toz H, et al. Long-term survival rates in haemodialysis patients treated with strict volume control. Nephrol Dial Transplant. 2006;21:3506-13. 